\title{
Auranofin: Repurposing an Old Drug for a Golden New Age
}

\author{
Christine Roder $\cdot$ Melanie J. Thomson
}

Published online: 20 February 2015

(c) The Author(s) 2015. This article is published with open access at Springerlink.com

\begin{abstract}
Drug discovery, development and registration is an expensive and time-consuming process associated with a high failure rate [Pessetto et al. (Mol Cancer Ther 12:1299-1309, 2013), Woodcock and Woosley (Annu Rev Med 59:1-12, 2008)]. Drug 'repurposing' is the identification of new therapeutic purposes for already approved drugs and is more affordable and achievable than novel drug discovery [Pessetto et al. (Mol Cancer Ther 12:1299-1309, 2013)]. Auranofin is a drug that is approved for the treatment of rheumatoid arthritis but is being investigated for potential therapeutic application in a number of other diseases including cancer, neurodegenerative disorders, HIV/AIDS, parasitic infections and bacterial infections [Tejman-Yarden et al. (Antimicrob Agents Chemother 57:2029-2035, 2013)]. The main mechanism of action of auranofin is through the inhibition of reduction/ oxidation (redox) enzymes that are essential for maintaining intracellular levels of reactive oxygen species. Inhibition of these enzymes leads to cellular oxidative stress and intrinsic apoptosis [Pessetto et al. (Mol Cancer Ther 12:1299-1309, 2013), Fan et al. (Cell Death Dis 5:e1191, 2014), Fiskus et al. (Cancer Res 74:2520-2532, 2014), Marzano et al. (Free Radic Biol Med 42:872-881, 2007)]. Drugs such as auranofin that have already been approved for human use [Tejman-Yarden et al. (Antimicrob Agents Chemother 57:2029-2035, 2013)] can be brought into clinical use for other diseases relatively quickly and for a fraction of the cost of new drugs.
\end{abstract}

C. Roder $(\varangle) \cdot$ M. J. Thomson

School of Medicine, Deakin University, Pigdons Road,

Waurn Ponds, VIC 3216, Australia

e-mail: cjrod@deakin.edu.au

\section{Key Points}

Drug 'repurposing' is more affordable and less timeconsuming than novel drug discovery.

Auranofin has potential as a therapy for a number of diseases including cancer, neurodegenerative disorders, HIV/AIDS, parasitic infections and bacterial infections.

\section{Introduction}

The current system of discovery, development and registration of new drugs is estimated to cost USD $\$ 1.5$ billion and often requires 10-17 years to complete [1]. This expensive and time-consuming process often results in failure, with an estimated 70-90\% of drugs failing clinical trials [2]. Drug 'repurposing' is the identification of new therapeutic applications for drugs that have received US FDA approval for another purpose. Due to the reduced length and cost of research and trial phases, drug repurposing is more affordable and achievable than novel drug discovery, with patients gaining access to new therapies more quickly [1].

One drug that is receiving increasing attention for its potential to be repurposed is Auranofin, approved for the treatment of rheumatoid arthritis in 1985 [3]. Auranofin [2,3,4,6-tetra- $o$-acetyl-L-thio- $\beta$-D-glycopyranp-sato- $S$-(triethyl-phosphine)-gold] has a well-known toxicity profile and is considered safe for human use [4-6]. It is a gold(I) compound with phosphine and thiol ligands in a linear arrangement (Fig. 1). After oral dosing, 15-25\% of 


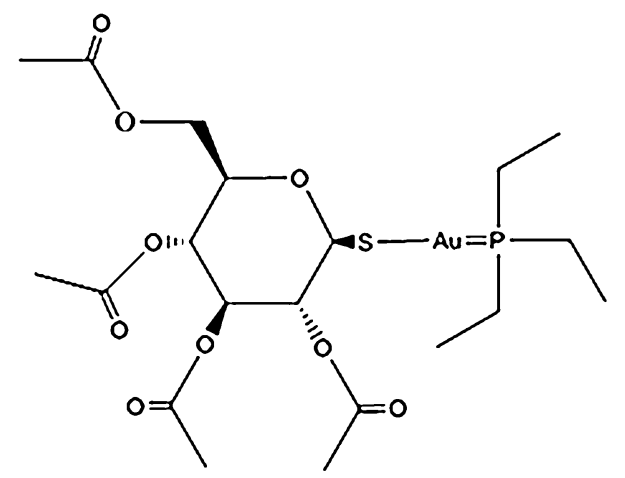

Fig. 1 The chemical structure of auranofin

the drug can be detected in the plasma, where it binds predominantly to albumin [6-8]. Most of this is absorbed via the gastrointestinal tract within the first $20 \mathrm{~min}$ [9], and within 1-2 $\mathrm{h}$ a peak plasma concentration of 6-9 $\mu \mathrm{g} /$ $100 \mathrm{~mL}$ is reached. The plasma half-life is $15-25$ days with almost total body elimination after 55-80 days [7, 8]. Auranofin is mainly excreted in the faeces $(85 \%)$, with only $15 \%$ appearing in the urine $[6,10]$. Just $0.4 \%$ of the administered dose is concentrated in the kidneys [6].

Rheumatoid arthritis is characterised by persistent inflammation and joint swelling leading to functional disability [6]. Auranofin was prescribed for the treatment of rheumatoid arthritis as a disease-modifying antirheumatic drug (DMARD) [6], and was able to slow disease progression by suppressing inflammation and stimulating cell-mediated immunity. Auranofin also inhibits phagocytosis by macrophages and the release of lysosomal enzymes and antibodies involved in cytotoxicity reactions [10, 11]. Although considered safer than the injectable gold counterparts (myochrysine, anochrysine, allochrysine and solganol) [6], aurnaofin was found to be less effective than other DMARDs, such as methotrexate, which led to a decline in the clinical use of auranofin [8]. Although DMARDs are still the primary treatment option for rheumatoid arthritis, there has been rapid development in biological therapies that inhibit cytokine targets, thus suppressing the pro-inflammatory cascade associated with rheumatoid arthritis. These drugs include tumour necrosis factor (TNF)$\alpha$ inhibitors such as the monoclonal antibodies infliximab, adalimumab, the TNF- $\alpha$-receptor fusion protein etanercept, and the interleukin (IL)-1 inhibitor anakinra [11].

The decline in the use of auranofin for the treatment of rheumatoid arthritis may be due, in part, to its adverse effects, most of which are associated with long-term use for chronic disease. The most common adverse effects are gastrointestinal complaints such as loose stools, abdominal cramping and watery diarrhoea, which can develop in the early months of treatment. The development of loose stools occurs in $40 \%$ of patients, while watery diarrhoea is reported in just $2-5 \%$ of patients, and in most cases these symptoms were alleviated by reducing or splitting the dose $[7,8]$. These symptoms are associated with changes in intestinal fluid movement and the net secretion of sodium, potassium, chloride and bicarbonate, as well as changes in the absorption of glucose and mannitol [12, 13]. Other adverse effects include skin irritations or rash, which occur in $20 \%$ of patients within the first year of treatment, as well as stomatitis and mouth ulcerations, which occur in $1-12 \%$ of patients and are often concomitant with skin rashes. Conjunctivitis occurs in $4 \%$ of patients, proteinuria occurs in up to $5 \%$ of patients, and there have been some reports of thrombocytopenia and bone marrow suppression, but these are extremely rare [7, 8].

Auranofin may no longer be the drug of choice for rheumatoid arthritis, but there is potential for new applications in the treatment of some cancers, parasitic infections, bacterial infections, HIV and even neurodegenerative disorders such as Parkinson's disease and Alzheimer's. Table 1 provides a list of studies investigating the repurposing of auranofin for diseases other than rheumatoid arthritis.

\section{Mechanisms of Action}

The main mechanism of action of auranofin is through the inhibition of reduction/oxidation (redox) enzymes such as thioredoxin reductase (TrxR). The thiol ligand contained in auranofin has a high affinity for thiol and selenol groups, to which it forms stable, and irreversible, adducts [14]. Redox enzymes such as TrxR are essential to many cellular processes, particularly in maintaining the intracellular levels of reactive oxygen species (ROS) [1, 14-16]. Controlling the level of ROS to prevent the resulting DNA damage is critical for the survival of all cell types, including cancer cells, parasites and memory $\mathrm{T}$ cells that harbour proviral HIV DNA. These particular cell types all over-express redox enzymes, which increases the affinity of auranofin towards these cells $[1,3,14-22]$. Inhibition of redox enzymes alters the redox state of the cell, which can lead to increased production of hydrogen peroxide and ROS that causes cellular oxidative stress and ultimately intrinsic apoptosis [1, 14-16].

Auranofin is particularly potent for selenoproteins and selenium-dependent enzymes because it also has a high affinity towards inorganic selenium in the form of selenide $\left(\mathrm{HSe}^{-}\right)$. Auranofin and $\mathrm{HSe}^{-}$are able to form a stable adduct through displacement of the sulphur in the auranofin thiol with the Se in the $\mathrm{HSe}^{-}$[23], resulting in a hydrogen sulphide by-product and the auranofin selenium compound. Selenoproteins such as TrxR in mammalian cells have been identified as a potential drug target for cancer [14], while 
Table 1 Diseases for which auranofin is being investigated as a potential treatment

\begin{tabular}{|c|c|}
\hline Disease & References \\
\hline \multicolumn{2}{|l|}{ Cancer } \\
\hline Leukaemia and lymphoma & {$[15,25,28,29]$} \\
\hline \multicolumn{2}{|l|}{ Chronic lymphocytic leukaemia (CLL) } \\
\hline \multicolumn{2}{|l|}{ Small lymphocytic lymphoma (SLL) } \\
\hline \multicolumn{2}{|l|}{ Prolymphocytic lymphoma (PLL) } \\
\hline \multicolumn{2}{|l|}{ Chronic myelogenous leukaemia (CML) } \\
\hline Human ovarian cancer (cisplatin-sensitive and cisplatin-resistant) & [16] \\
\hline Gastrointestinal stromal tumour (GIST; imatinib-resistant) & {$[14]$} \\
\hline \multicolumn{2}{|l|}{ Neurodegenerative disorders } \\
\hline Alzheimer's and Parkinson's disease & {$[5,9]$} \\
\hline HIV/AIDS & {$[21,31]$} \\
\hline \multicolumn{2}{|l|}{ Parasitic infections } \\
\hline Trypanosomatids & {$[17,19,33]$} \\
\hline \multicolumn{2}{|l|}{ Trypanosoma spp. } \\
\hline \multicolumn{2}{|l|}{ Leishmania spp. } \\
\hline Platyhelminthes & {$[22,34,35]$} \\
\hline \multicolumn{2}{|l|}{ Schistosoma spp. } \\
\hline \multicolumn{2}{|l|}{ Echinococcus granulosus } \\
\hline Giardia lamblia & {$[3,18]$} \\
\hline Enamoeba histolytica & [18] \\
\hline Apicomplexa & {$[4,36]$} \\
\hline \multicolumn{2}{|l|}{ Toxoplasmosa gondii } \\
\hline \multicolumn{2}{|l|}{ Plasmodium falciprum } \\
\hline \multicolumn{2}{|l|}{ Bacterial infections } \\
\hline Staphylococcus aureas & [42] \\
\hline Clostridium difficile & {$[23]$} \\
\hline Treponema denticola & {$[24]$} \\
\hline Enterococcus faecalis & {$[43]$} \\
\hline
\end{tabular}

selenoproteins involved in Stickland reactions in amino acid-fermenting bacteria have been identified as a potential drug target for these bacterial infections [23, 24].

An alternate mechanism of action of auranofin is through inhibiting the ubiquitin-proteasome system (UPS) in cancer cells. Studies have shown that many different cancers, such as colon, prostate and leukaemia, rely on the UPS system more heavily than non-cancer cells [25, 26]. The UPS system is involved in many cellular processes, including cell cycle regulation, protein degradation, gene expression and DNA repair. The $26 \mathrm{~S}$ proteasome consists of two parts: the $20 \mathrm{~S}$ proteasome peptidase and the $19 \mathrm{~S}$ proteasome-associated deubiquitinase (DUBs) [25, 26]. Liu et al. [26] reported that inhibiting either part of the $26 \mathrm{~S}$ proteasome induced apoptosis, and that the current treatment for relapsed myeloma and mantle cell lymphoma, bortezomib/Velcade ${ }^{\circledR}$, targeted the 20S proteasome peptidase. Auranofin, on the other hand, inhibited the DUBs, suggesting that these two drugs inhibited UPS by different mechanisms. The authors suggested that the activity of auranofin may be due to its $\mathrm{Au}-\mathrm{S}$ bond, shown in Fig. 1, as blocking this active site prevents auranofin from inhibiting DUBs and inducing apoptosis.

\section{Cancer}

Cancer is a major public health problem in many parts of the world, with the estimated number of new cases in the USA every year in excess of 1,600,000 [27]. Although death rates in the USA are declining by just over $1 \%$ annually, there are still more than 500,000 deaths [27]. The development of resistance to the current first-line chemotherapeutics by cancer cells is one of the major reasons for clinical failure of therapy and has prompted the search for new anti-cancer therapies. Auranofin is effective against cancer cells by two mechanisms of action. The first is through inhibition of mammalian TrxR (mTrxR), which is a critical regulator of redox balance in the cytosol and in the mitochondria. Auranofin is a potent inhibitor of mTrxR because of the presence of selenium in the form of selenocysteine in mTrxR [1,14-16]. The second is through 
inhibition of the UPS system by targeting DUBs, which are involved in cell cycle regulation, protein degradation, gene expression and DNA repair $[25,26]$. Both these mechanisms of action induce apoptosis.

In 1985, Mirabelli et al. [28] showed that auranofin had potent in vitro cytotoxic activity against a number of tumour cell lines including P388 mouse leukaemia. They went on to demonstrate that auranofin also had in vivo activity against P388 leukaemia with a maximum cell kill of $0.6 \mathrm{log}$ at a single daily dose of $8 \mathrm{mg} / \mathrm{kg}$ given via intraperitoneal injection. Since this time there have been numerous in vitro and in vivo studies focusing on the antitumour activity of auranofin. Marzano et al. [16] were able to show that auranofin induced apoptosis in vitro in both cisplatin-sensitive (2008) and cisplatin-resistant (C13*) human ovarian cancer cells, observing that auranofin is more effective at decreasing cell viability than cisplatin. Pessetto et al. [1] have also demonstrated the apoptotic activity of auranofin against tumour cells in vitro, this time in gastrointestinal stromal tumour (GIST) cells (GIST-T1), including imatinib-resistant GIST. There have also been studies focusing on chronic lymphocytic leukaemia (CLL), which is associated with high rates of relapse and resistance to treatments. Fiskus et al. [15] reported that auranofin showed apoptotic activity against cultured and patient-derived CLL cells. Auranofin is currently in a phase I/II clinical trial for the treatment of CLL, small lymphocytic lymphoma (SLL) and prolymphocytic lymphoma (PLL) (NCT01419691) [29]. Fan et al. [14] not only demonstrated the in vitro and in vivo apoptotic activity of auranofin against non-small-cell lung cancer (A549 human lung adenocarcinoma) but also investigated the enhancement of this activity through the addition of selenocysteine, a natural inhibitor of TrxR, theorising that selenocysteine competes with thioredoxin, the substrate for TrxR.

While investigating its other mechanism of action against cancer cells, Chen et al. demonstrated that auranofin had a strong cytotoxic effect against chronic myelogenous leukaemia (CML) expressing the fusion oncoprotein Bcr-Abl. Further to this they showed that auranofin could overcome resistance to the tyrosine kinase inhibitor imatinib mesylate, which is the current treatment for chronic-phase CML. Resistance to imatinib mesylate is caused by point mutations in the $B c r$-Abl gene, and while new tyrosine kinase inhibitors such as nolotinib, dasatinib and INNO-406 are effective against most of these mutations, they are not effective against the most common, a T315I missense mutation which accounts for $20 \%$ of all point mutations of $B c r-A b l$. Auranofin is able to inhibit expression of $B c r-A b l$ and induce caspase activation that cleaves Bcr-Abl, leading to downregulation of $B c r-A b l$ and reduced cell proliferation [25]. Independent of this, auranofin also induces apoptosis by inhibiting DUBs [25, 26].

\section{Neurodegenerative Disorders}

Neurodegenerative disorders such as Alzheimer's and Parkinson's disease have both been linked to inflammation in the CNS $[5,9]$. This inflammation is driven by the activation of the glial cells, microglia and astrocytes, which when activated release neurotoxins and inflammatory mediators. When combined with a deficiency in neurotrophic factors, these neurotoxins and inflammatory mediators can harm the nearby neurons, contributing to progression of neurodegenerative disorders [5, 9]. Reducing the inflammation, decreasing the release of neurotoxins or increasing the release of neurotrophic factors could reduce neuronal loss, potentially slowing the progression of these diseases [5, 9]. Current treatments for neurodegenerative disorders include antioxidants and metal-protein attenuating compounds (MPACs) such as desferrioxamine for Alzheimer's disease. These treatments target the abnormal metal-protein interactions and the associated oxidative stress that also contribute to neuron degeneration [30], but not the inflammation driven by activated glial cells [5].

Auranofin acts as an anti-inflammatory, altering cytokine levels by increasing IL-8 and reducing IL-6 secretion from lipopolysaccharide-stimulating human monocytes. Auranofin also induces the anti-inflammatory enzyme heme oxygenase (HOX)- 1 in the human monocyte cell line THP-1 cells, protecting neuronal cells from oxidative stress induced by hydrogen peroxide [5]. In addition to this, Madeira et al. [5] demonstrated that auranofin can inhibit the neurotoxic effects of stimulated primary human astrocytes and U-373 astrocyte cells toward human neuronal cells in low micromolar concentrations $(0.1-5 \mu \mathrm{mol} / \mathrm{L})$. They then demonstrated that auranofin can also inhibit the neuronal cells from the microglia toxins TNF- $\alpha$ and nitric oxide [9]. The authors suggested that auranofin may have neuroprotective activity in addition to the previously described anti-inflammatory activity [5, 9], although the mechanisms for this are not known. Auranofin has been shown to reach the CNS in low micromolar concentrations $(0.2-5 \mu \mathrm{mol} / \mathrm{L})$, indicating that enough auranofin can cross the blood-brain barrier to have similar in vivo protective effects to those seen in vitro [9].

\section{HIV/AIDS}

Complete elimination of HIV is a distant goal, with current treatment goals being a functional cure that will effectively control the virus within the host. An ideal functional cure is one that is able to be withdrawn for prolonged periods without risk of the virus rebounding to pre-therapy levels [31]. People with HIV/AIDS need lifelong antiretroviral (ARV) therapy as HIV infection will persist despite this 
treatment, with viral titres increasing upon withdrawal. There are also viral reservoirs that are invulnerable to ARV therapy, such as the central and transitional memory T cells (TCM and TTM, respectively) [21, 31]. TCM and TTM are long-lived and carry dormant proviral DNA copies of the virus integrated into the genome. These DNA copies cannot be targeted by either the immune system or current drug-based therapies. Auranofin has been shown to induce cell death in TCM and TTM [21]. Studies using combined ARV and auranofin therapy in rhesus macaques infected with the HIV simian homologue SIVmac251 have been able to maintain the reduced viral titre long-term after treatment was withdrawn. The effects of auranofin on the memory $\mathrm{T}$ cells were not associated with any detectable immune impairment [21]. Another study, also using the macaque primate model, added buthionine sulfoimine (BSO) to the ARV/auranofin therapy, which is an inhibitor of glutathione synthesis. This combination therapy resulted in long-term reduction in the post-viral set point larger than that of the ARV/auranofin therapy described above [31].

Auranofin is able to induce cell death in TCM and TTM in the same way it does in cancer cells and some parasites, by inhibiting TrxR activity. Inhibition of TrxR leads to increased oxidative stress in the cells, which activates the redox-sensitive apoptosis pathways [21]. BSO inhibits the synthesis of glutathione, another intracellular antioxidant. Both auranofin and BSO have been shown to induce partially selective killing of infected cells in vitro [31]. The combination of two drugs that induce redox-sensitive cell death by targeting different cellular targets could ensure more widespread death of HIV-infected memory $\mathrm{T}$ cells, resulting in longer periods of reduced viral titre following withdrawal of treatment.

\section{Parasitic Infections}

Many parasitic infections are classified as Neglected Tropical Diseases (NTDs) by the Centers for Disease Control and Prevention (CDC) as there is a lack of attention by the global public health community [32]. NTDs affect onesixth of the world's population [32], with malaria (Plasmodium falciparum) alone killing approximately 660,000 people each year [22]. NTDs are more prevalent among the world's poorest peoples, who often have limited access to adequate healthcare. Further compounding this problem is the development of drug resistance to the current treatments [32]. Clearly there is a need for alternative therapies that are both inexpensive and easily attainable.

Like cancer cells, parasites need to maintain cellular redox balance, and rely on redox enzymes such as TrxR, the thioredoxin-glutathione reductase system (TGR) and typanthione reductase (TypR) to prevent an accumulation of hydrogen peroxide and ROS in the cytosol and mitochondria. Auranofin is able to inhibit the action of the TrxR, TGR or TypR enzymes, hence allowing oxidative stress to damage the parasites [3, 17-20, 22]. The effects of auranofin have been studied on a number of parasitic species including Giardia lamblia [3, 18], Entamoeba histolytica [18], Trypanosoma brucei [33], Leishmania infantum [17, 19], L. major [19], Schistosoma mansoni [22, 34, 35], Echinococcus granulosus [34], Toxoplasmsa gondii [36] and P. falciparum [2, 4].

Debnath et al. [18] investigated the effects of auranofin on G. lamblia and E. histolytica, which cause the diarrhoeal diseases giardia and amebasis, respectively. There are currently no vaccines or prophylactics for either of these diseases, and first-line therapy is usually nitramidazoles such as metronidazole. E. histolytica trophozites are able to adapt to therapeutic concentrations of metronidazole and there is clinical evidence for metronidazole resistance in G. lamblia. Debnath et al. [18] demonstrated that E. histolytica trophozites are more sensitive to killing by hydrogen peroxide and ROS when exposed to auranofin due to inhibition of TrxR. Similar results have been described for G. lamblia in both this study [3] and another by Tejman-Yarden et al., who investigated the effects of auranofin on metronidazole-resistant G. lamblia [18].

Trypanosomatids are an order of protozoan parasites responsible for diseases such as sleeping sickness, South American Chagas disease and leishmaniasis [17, 33]. Sleeping sickness and Chagas disease are caused by Trypanosoma spp. Annually 300,000-500,000 people are infected with sleeping sickness and 40,000 people die from it, while Chagas disease affects 16-18 million people [33]. Leishmaniasis is caused by Leishmania spp. and is endemic in 98 countries with approximately 2 million new cases annually [17]. Treatment for sleeping sickness depends on the stage of the disease. First-stage treatments such as pentamidine and suramin have a relatively lower toxicity and are easier to administer. Second-stage drugs include melarsoprol, which is fatal in 3-10 \% of cases, and eflornethine, which is less toxic than melarsoprol but requires a strict and difficult to apply regimen and is only effective against Trypanosoma brucei gambiense, not T. b. rhodesiense [37]. Chagas disease is treated with benznidazole or nifurtimox, which are both $100 \%$ effective for the acute phase of the disease, but less so for the chronic phase [38]. Treatment for leishmaniasis can range from sodium stibogluconate and amphotericin B to miltefosin and paromomycin; however, these treatments have detrimental effects or pharmacological liabilities that result in treatment failure or relapse of disease. There is no optimal therapy and the response to any of these drugs is inconsistent. As a result, many cases of leishmaniasis go untreated, leaving the patient disfigured due to the characteristic lesions caused by 
these parasites [17]. Lobanov et al. reported high rates of conservation of selenoproteins in Trypanosoma and Leishmania species. They also demonstrated that auranofin was highly toxic for both bloodstream and procyclic stages of $T$. b. brucei, concluding that inhibiting redox selenoproteins may be effective against these types of infections [33]. Leishmania spp. utilise a typanothione-based system to reduce oxidative stress and to synthesise and utilise spermidine, which is essential for the parasites' growth and survival [19]. Ilari et al. [19] demonstrated that auranofin could induce a dose-dependent anti-proliferative effect on L. infantum and L. major promastigotes. Similar results were found by Sharlow et al. [17], who studied the effect of auranofin on L. major and L. amazonensis. Both of these studies investigated the ability of auranofin to inhibit TypR, leading to an apoptotic-like response in promastigotes $[17,19]$.

Parasites from the phylum Playhelminth are responsible for diseases such as schistomanasis and hydatid. The current treatment for these infections is praziquantel, which is still successful in most cases [34]. Continued use of praziquantel has seen the emergence of drug resistance, which is of serious concern since it is the only drug that is readily available for large-scale treatment of these diseases [22, 34]. Platyelminths such as Schistosoma mansoni and Echinciccus granulosis rely on TGR to prevent oxidative stress caused by ROS [22]. Studies by Kuntz et al. [35], Bonilla et al. [34] and Caroli et al. [22] have shown that auranofin can induce oxidative stress and inhibit growth in both these organisms by inhibiting the activity of TGR.

$P$. falciparum causes malaria, a blood infection that kills 660,000 people annually [22]. The treatment for malaria is chloroquine, but there has been an emergence of resistance to this drug [22]. P. falciparum relies on a TrxR and a glutathione reductase to maintain redox homeostastis [4, 22]. Auranofin has been shown to inhibit the activity TrxR in $P$. falciparum, leading to severe oxidative stress and inhibition of growth [4]. Another parasite from the phylum Apicomplexa is Toxoplasma gondii, which affects an estimated two-thirds of the world population. There are very few treatments against this parasite, most of which are only active against the active lifecycle stage (tachyozoite) and are unable to eradicate the parasite from the human body, leaving the host susceptible to recurrence. Infection with $T$. gondii can cause long-term complications such as blindness or neurological abnormalities, due to the parasite's fondness for the brain and retinas [36]. Auranofin has been shown to reduce parasitic replication in vitro and parasitic load in vivo. In a chicken embryo model of acute toxoplasmosis, a single dose of $1 \mathrm{mg} / \mathrm{kg}$ of estimated body weight was able to prevent death. Although the authors of this study are yet to determine the exact molecular target, they do speculate that auranofin acts on one of the multiple antioxidant enzymes used to maintain redox homeostasis in T. gondii [36].

\section{Bacterial Infections}

Antibacterial (antibiotic) resistance is one of the greatest public health crises facing us today. Without effective antibacterials it will not be possible to care for premature infants or the critically ill, cancer patients would not be able to receive chemotherapy and many surgeries, especially organ transplants, would carry too high a risk of untreatable infection [39]. Although there is an urgent need for novel antimicrobials, pharmaceutical companies are withdrawing from the antibacterial market, putting antibacterial development at a standstill [39]. It makes sense to repurpose old drugs with potential antimicrobial activity for the treatment of antibacterial-resistant infections. Auranofin has shown antimicrobial activity against a number of bacterial pathogens including Staphylococcus aureas, Clostridium difficile, Treponema denticola and Enterococcus faecalis.

C. difficile is a Gram-positive, anaerobic hospital-acquired infection (HAI), the symptoms of which range from diarrhoea to severe and life-threatening pseudomembranous colitis [23]. The ability of this pathogen to cause widespread disease coupled with its potential for antibacterial resistance prompted the CDC to raise its threat level to urgent [40]. T. denticola is one of the primary pathogens responsible for periodontitis, one of the major causes of adult tooth loss. Both of these bacteria rely on Stickland reactions performed by the selenoprotein glycine reductase for energy. Auranofin is able to inhibit the activity of glycine reductase by forming a stable bond with inorganic selenium, disrupting selenium metabolism and the synthesis of selenocysteine. Without selenocysteine, these bacteria cannot synthesise glycine reductase, and are therefore unable to reduce glycine for energy production [23, 24]. C. difficile also uses the selenoprotein proline reductase for energy production. The activity of proline reductase is also inhibited by auranofin in a similar fashion to glycine reductase [41]. Auranofin has been shown to inhibit the growth of $C$. difficile and $T$. denticola in vitro at low micromolar concentrations [23, 24].

Methicillin-resistant $S$. aureas (MRSA) infections are responsible for over 80,000 infections annually in the USA [40]. The first-line treatment for MRSA is vancomycin, but with the emergence of vancomycin-resistant $S$. aureas (VRSA) there is an urgent need for another alternative antibacterial. Auranofin has shown activity against $S$. aureas in the nanomolar range (150-300 nM), including activity against both MRSA and VRSA. The mechanism of growth inhibition is not yet known [42]. 
E. faecalis is an HAI that produces biofilms during infection, usually in the bladder as the result of catherisation, but can also produce vegetative growths on heart tissue [43]. Vancomycin-resistant Enterococcus spp. (VRE) are a serious threat to public health, as there are very few treatment options left after vancomycin. E. faecalis infections make up approximately $9 \%$ of the 20,000 VRE HAIs seen annually in the USA [40]. Biofilm production in $E$. faecalis has been linked to a putative xanthine dehydrogenase (EF2570), a labile selenoprotein that is upregulated in the presence of uric acid, selenium and molybdenum. There is evidence that this xanthine dehydrogenate is necessary for extracellular superoxide and hydrogen peroxide production, which correlated with biofilm density. Reducing selenium bioavailability inhibits the activity of xanthine dehydrogenase, resulting in a reduction in biofilm density. Auranofin was able to inhibit the biofilm formation of E. faecalis at a concentration of $1 \mu \mathrm{mol} / \mathrm{L}$ [43].

\section{Summary}

Drug repurposing can provide new therapeutic options for a vast number of diseases where current therapies are failing or are inadequate. Multidrug resistance is becoming problematic in diseases such as cancer, parasitic infections and bacterial infections, leading to poor clinical outcomes. Current options for diseases such as HIV require ongoing therapy, while the treatment options for neurodegenerative disorders such as Alzheimer's and Parkinson's disease target metal homeostasis and related oxidative stress but not the inflammation.

Auranofin has the potential to be repurposed for a lot of diseases. Auranofin has a high affinity for thiols and selenoproteins and is able to inhibit enzymes that reduce intracellular ROS such as TrxR, which have been shown to be an effective therapeutic target for cancer, parasitic infections and HIV. The high affinity of auranofin to selenium and selenoproteins disrupts bacterial selenium metabolism, a potential therapeutic target for the hospital-acquired $C$. difficile infection and drug-resistant infections such as MRSA and VRE. Further to this, auranofin has been described as having antiinflammatory and neuroprotective activity that could prove useful in the treatment of neurodegenerative disorders.

New drug discovery is an expensive and risky endeavour. Coupled with reduced funding and support from government and industry bodies, particularly in the areas of antimicrobials and NTDs, the drug discovery pipeline is slowing down. It makes sense to look to old drugs that have already passed clinical trials and received FDA approval for other diseases. These drugs can be brought into clinical use for other diseases relatively quickly and for a fraction of the cost of new drugs.
Acknowledgments The authors have no conflict of interest to declare.

Open Access This article is distributed under the terms of the Creative Commons Attribution Noncommercial License which permits any noncommercial use, distribution, and reproduction in any medium, provided the original author(s) and the source are credited.

\section{References}

1. Pessetto ZY, Weir SJ, Sethi G, Broward MA, Godwin AK. Drug repurposing for gastrointestinal stromal tumor. Mol Cancer Ther. 2013;12(7):1299-309. doi:10.1158/1535-7163.mct-12-0968.

2. Woodcock J, Woosley R. The FDA critical path initiative and its influence on new drug development. Annu Rev Med. 2008;59:1-12.

3. Tejman-Yarden N, Miyamoto Y, Leitsch D, Santini J, Debnath A, Gut $\mathrm{J}$, et al. A reprofiled drug, auranofin, is effective against metronidazole-resistant Giardia lamblia. Antimicrob Agents Chemother. 2013;57(5):2029-35.

4. Sannella AR, Casini A, Gabbiani C, Messori L, Bilia AR, Vincieri FF, et al. New uses for old drugs. Auranofin, a clinically established antiarthritic metallodrug, exhibits potent antimalarial effects in vitro: mechanistic and pharmacological implications. FEBS Lett. 2008;582(6):844-7.

5. Madeira JM, Renschler CJ, Mueller B, Hashioka S, Gibson DL, Klegeris A. Novel protective properties of auranofin: inhibition of human astrocyte cytotoxic secretions and direct neuroprotection. Life Sci. 2013;92(22):1072-80.

6. Messori L, Marcon G. Gold complexes in the treatment of rheumatoid arthritis. Met Ions Biol Syst. 2004;41:279-304.

7. Kean WF, Kean IR. Clinical pharmacology of gold. Inflammopharmacology. 2008;16(3):112-25.

8. Kean WF, Hart L, Buchanan WW. Auranofin. Br J Rheumatol. 1997;36(5):560-72.

9. Madeira JM, Bajwa E, Stuart MJ, Hashioka S, Klegeris A. Gold drug auranofin could reduce neuroinflammation by inhibiting microglia cytotoxic secretions and primed respiratory burst. J Neuroimmunol. 2014;276(1-2):71-9.

10. Walz DT, DiMartino MJ, Griswold DE, Intoccia AP, Flanagan TL. Biologic actions and pharmacokinetic studies of auranofin. Am J Med. 1983;75(6A):90-108.

11. Doan T, Massarotti E. Rheumatoid arthritis: an overview of new and emerging therapies. J Clin Pharmacol. 2005;45(7):751-62.

12. Behrens R, Devereaux M, Hazleman B, Szaz K, Calvin J, Neale G. Investigation of auranofin-induced diarrhoea. Gut. 1986;27(1): $59-65$.

13. Ammon HV, Fowle SA, Cunningham JA, Komorowski RA, Loeffler RF. Effects of auranofin and myochrysine on intestinal transport and morphology in the rat. Gut. 1987;28(7):829-34.

14. Fan C, Zheng W, Fu X, Li X, Wong YS, Chen T. Enhancement of auranofin-induced lung cancer cell apoptosis by selenocystine, a natural inhibitor of TrxR1 in vitro and in vivo. Cell Death Dis. 2014;5:e1191.

15. Fiskus W, Saba N, Shen M, Ghias M, Liu J, Gupta SD, et al. Auranofin induces lethal oxidative and endoplasmic reticulum stress and exerts potent preclinical activity against chronic lymphocytic leukemia. Cancer Res. 2014;74(9):2520-32.

16. Marzano C, Gandin V, Folda A, Scutari G, Bindoli A, Rigobello MP. Inhibition of thioredoxin reductase by auranofin induces apoptosis in cisplatin-resistant human ovarian cancer cells. Free Radic Biol Med. 2007;42(6):872-81.

17. Sharlow ER, Leimgruber S, Murray S, Lira A, Sciotti RJ, Hickman M, et al. Auranofin is an apoptosis-simulating agent 
with in vitro and in vivo anti-leishmanial activity. ACS Chem Biol. 2014;9(3):663-72.

18. Debnath A, Ndao M, Reed SL. Reprofiled drug targets ancient protozoans. Gut Microbes. 2013;4(1):66-71.

19. Ilari A, Baiocco P, Messori L, Fiorillo A, Boffi A, Gramiccia M, et al. A gold-containing drug against parasitic polyamine metabolism: the X-ray structure of trypanothione reductase from Leishmania infantum in complex with auranofin reveals a dual mechanism of enzyme inhibition. Amino Acids. 2012;42(2-3): 803-11.

20. Watkins RR, Eckmann L. Treatment of giardiasis: current status and future directions. Curr Infect Dis Rep. 2014;16(2):396.

21. Chirullo B, Sgarbanti R, Limongi D, Shytaj IL, Alvarez D, Das B, et al. A candidate anti-HIV reservoir compound, auranofin, exerts a selective 'anti-memory' effect by exploiting the baseline oxidative status of lymphocytes. Cell Death Dis. 2013;4:e944.

22. Caroli A, Simeoni S, Lepore R, Tramontano A, Via A. Investigation of a potential mechanism for the inhibition of SmTGR by auranofin and its implications for Plasmodium falciparum inhibition. Biochem Biophys Res Commun. 2012;417(1):576-81.

23. Jackson-Rosario S, Cowart D, Myers A, Tarrien R, Levine RL, Scott RA, et al. Auranofin disrupts selenium metabolism in Clostridium difficile by forming a stable Au-Se adduct. J Biol Inorg Chem. 2009;14(4):507-19.

24. Jackson-Rosario S, Self WT. Inhibition of selenium metabolism in the oral pathogen Treponema denticola. J Bacteriol. 2009; 191(12):4035-40.

25. Chen X, Shi X, Zhao C, Li X, Lan X, Liu S, et al. Anti-rheumatic agent auranofin induced apoptosis in chronic myeloid leukemia cells resistant to imatinib through both Bcr/Abl-dependent and independent mechanisms. Oncotarget. 2014;5(19):9118-32.

26. Liu N, Li X, Huang H, Zhao C, Liao S, Yang C, et al. Clinically used antirheumatic agent auranofin is a proteasomal deubiquitinase inhibitor and inhibits tumor growth. Oncotarget. 2014;5(14): 5453-71.

27. Siegel R, Naishadham D, Jemal A. Cancer statistics, 2012. CA Cancer J Clin. 2012;62(1):10-29.

28. Mirabelli CK, Johnson RK, Sung CM, Faucette L, Muirhead K, Crooke ST. Evaluation of the in vivo antitumor activity and in vitro cytotoxic properties of auranofin, a coordinated gold compound, in murine tumor models. Cancer Res. 1985;45(1): $32-9$.

29. University of Kansas. Phase I and II study of auranofin in chronic lymphocytic leukemia (CLL). [ClinicalTrials.gov identifier NCT01419691]. US National Institutes of Health, ClinicalTrials.gov. http://clinicaltrials.gov.

30. Barnham KJ, Masters CL, Bush AI. Neurodegenerative diseases and oxidative stress. Nat Rev Drug Discov. 2004;3(3):205-14.

31. Shytaj IL, Chirullo B, Wagner W, Ferrari MG, Sgarbanti R, Corte $\mathrm{AD}$, et al. Investigational treatment suspension and enhanced cell-mediated immunity at rebound followed by drug-free remission of simian AIDS. Retrovirology. 2013;10(1):71.

32. CDC's neglected tropical disease program. Atlanta: Center for Global Health, Division of Parasitic diseases and Malaria; 2014. http://www.cdc.gov/globalhealth/ntd/resources/ntd_factsheet.pdf. Accessed 2 Feb 2015.

33. Lobanov AV, Gromer S, Salinas G, Gladyshev VN. Selenium metabolism in Trypanosoma: characterization of selenoproteomes and identification of a Kinetoplastida-specific selenoprotein. Nucleic Acids Res. 2006;34(14):4012-24.

34. Bonilla M, Denicola A, Novoselov SV, Turanov AA, Protasio A, Izmendi $\mathrm{D}$, et al. Platyhelminth mitochondrial and cytosolic redox homeostasis is controlled by a single thioredoxin glutathione reductase and dependent on selenium and glutathione. J Biol Chem. 2008;283(26):17898-907.

35. Kuntz AN, Davioud-Charvet E, Sayed AA, Califf LL, Dessolin J, Arner ES, et al. Thioredoxin glutathione reductase from Schistosoma mansoni: an essential parasite enzyme and a key drug target. PLoS Med. 2007;4(6):e206.

36. Andrade RM, Chaparro JD, Capparelli E, Reed SL. Auranofin is highly efficacious against Toxoplasma gondii in vitro and in an in vivo experimental model of acute toxoplasmosis. PLoS Negl Trop Dis. 2014;8(7):e2973.

37. World Health Organisation (WHO). Trypanosomiasis, human African (sleeping sickness). Fact sheet no. 259. 2014. http://www. who.int/mediacentre/factsheets/fs259/en/. Accessed 14 Jan 2015.

38. World Health Organisation (WHO). Chagas disease (American trypanosomiasis). Fact sheet no. 340. 2014. http://www.who.int/ mediacentre/factsheets/fs340/en/. Accessed 14 Jan 2015.

39. Infectious Diseases Society of America. Combating antimicrobial resistance: policy recommendations to save lives. Clin Infect Dis. 2011;52(suppl 5):S397-428.

40. Antibiotic resistant threats in the United States. US Department of Health and Human Services, Centers for Disease Control and Prevention. 2013. http://www.cdc.gov/drugresistance/threatreport2013/pdf/ar-threats-2013-508.pdf. Accessed 27 Nov 2014.

41. Jackson S, Calos M, Myers A, Self WT. Analysis of proline reduction in the nosocomial pathogen Clostridium difficile. J Bacteriol. 2006;188(24):8487-95.

42. Hokai Y, Jurkowicz B, Fernandez-Gallardo J, Zakirkhodjaev N, Sanau M, Muth TR, et al. Auranofin and related heterometallic gold(I)-thiolates as potent inhibitors of methicillin-resistant Staphylococcus aureus bacterial strains. J Inorg Biochem. 2014; 138C:81-8.

43. Srivastava M, Mallard C, Barke T, Hancock LE, Self WT. A selenium-dependent xanthine dehydrogenase triggers biofilm proliferation in Enterococcus faecalis through oxidant production. J Bacteriol. 2011;193(7):1643-52. 